\title{
The Dopaminergic Midbrain Participates in Human Episodic Memory Formation: Evidence from Genetic Imaging
}

\author{
Björn H. Schott, ${ }^{1,3}$ Constanze I. Seidenbecher, ${ }^{3}$ Daniela B. Fenker, ${ }^{1}$ Corinna J. Lauer, ${ }^{1}$ Nico Bunzeck, ${ }^{1}$ \\ Hans-Gert Bernstein, ${ }^{2}$ Wolfgang Tischmeyer, ${ }^{3}$ Eckart D. Gundelfinger, ${ }^{3}$ Hans-Jochen Heinze, ${ }^{1,4}$ and Emrah Düzel ${ }^{1,5}$ \\ ${ }^{1}$ Department of Neurology II and Center for Advanced Imaging and 2Department of Psychiatry, University Hospital of Magdeburg, 39120 Magdeburg, \\ Germany, Departments of ${ }^{3}$ Neurochemistry and Molecular Biology and ${ }^{4}$ Behavioral Neurology, Leibniz-Institute for Neurobiology, 39118 Magdeburg, \\ Germany, and 5 Institute for Cognitive Neuroscience, and National Hospital for Neurology and Neurosurgery, University College London, London WC1N \\ 3AR, United Kingdom
}

Recent data from animal studies raise the possibility that dopaminergic neuromodulation promotes the encoding of novel stimuli. We investigated a possible role for the dopaminergic midbrain in human episodic memory by measuring how polymorphisms in dopamine clearance pathways affect encoding-related brain activity (functional magnetic resonance imaging) in an episodic memory task. In 51 young, healthy adults, successful episodic encoding was associated with activation of the substantia nigra. This midbrain activation was modulated by a functional variable number of tandem repeat (VNTR) polymorphism in the dopamine transporter (DAT1) gene. Despite no differences in memory performance between genotype groups, carriers of the (low expressing) 9-repeat allele of the DAT1 VNTR showed relatively higher midbrain activation when compared with subjects homozygous for the 10-repeat allele, who express DAT1 at higher levels. The catechol-O-methyl transferase (COMT) Val108/158Met polymorphism, which is known to modulate enzyme activity, affected encoding-related activity in the right prefrontal cortex (PFC) and in occipital brain regions but not in the midbrain. Moreover, subjects homozygous for the (low activity) Met allele showed stronger functional coupling between the PFC and the hippocampus during encoding. Our finding that genetic variations in the dopamine clearance pathways affect encoding-related activation patterns in midbrain and PFC provides strong support for a role of dopaminergic neuromodulation in human episodic memory formation. It also supports the hypothesis of anatomically and functionally distinct roles for DAT1 and COMT in dopamine metabolism, with DAT1 modulating rapid, phasic midbrain activity and COMT being particularly involved in prefrontal dopamine clearance.

Key words: dopamine transporter; catechol-0-methyl transferase; polymorphism; dopamine; midbrain; episodic memory; fMRI

\section{Introduction}

Episodic memory (Tulving, 2002), the ability to encode and recall personal events, is critically dependent on the hippocampus (Vargha-Khadem et al., 1997; Egan et al., 2003; Squire et al., 2004), and functional neuroimaging studies have shown that episodic memory encoding is associated with activations of the hippocampus and its interactions with the prefrontal cortex (PFC) (Gabrieli et al., 1997; Buckner et al., 2000; Tulving, 2002).

A role for dopamine in working memory has been shown in patients and healthy subjects (Egan et al., 2001; Mattay et al., 2002; Aalto et al., 2005), and there is increasing evidence for a role of dopamine in episodic memory (Lisman and Grace, 2005). Dopamine can promote long-term potentiation, a widely accepted

Received May 13, 2005; revised Dec. 13, 2005; accepted Dec. 13, 2005.

This work was supported by the Deutsche Forschungsgemeinschaft (Sonderforschungsbereich 426, TP D1 and A1), the International Leibniz Program (B.H.S., D.B.F.), the German National Academic Foundation (C.J.L.), The Helmholtz Gemeinschaft (Virtual Institute VH-VI-125), and the Fonds der Chemischen Industrie (E.D.G.). We thank Ulrike Malecki, Adrienn Zsóri, Kerstin Möhring, Ilona Wiedenhöft, and Jörn Kaufmann for assistance with MRI scanning and analysis, Grazyna Debska-Vielhaber for help with genotyping, and Anne Braun, Daniel Fischer, and Karl-Heinz Smalla for help with protein biochemistry.

Correspondence should be addressed to Björn H. Schott, Klinik für Neurologie Il, Otto von Guericke Universität Magdeburg, Leipziger Strasse 44, 39120 Magdeburg, Germany. E-mail: bschott@neuro2.med.uni-magdeburg.de. DOI:10.1523/JNEUROSCI.3463-05.2006

Copyright $\odot 2006$ Society for Neuroscience $\quad$ 0270-6474/06/261407-11\$15.00/0 model of hippocampus-dependent memory (Otmakhova and Lisman, 1996; Li et al., 2003), and improve hippocampusdependent learning in animals (Gasbarri et al., 1996; Bach et al., 1999; Schröder et al., 2003). In humans, pathological alterations in the dopaminergic system are often accompanied by memory deficits (van Oostrom et al., 2003). The dopamine precursor L-3,4-dihydroxyphenylalanine (L-dopa) enhances memory performance (Knecht et al., 2004), and striatal dopamine transporter availability correlates positively with learning performance (Mozley et al., 2001).

Anatomical evidence for a role of the dopaminergic midbrain in episodic encoding comes from function magnetic resonance imaging (fMRI). Reward-related activation of the medial substantia nigra is associated with improved hippocampus-dependent memory consolidation (Wittmann et al., 2005). Encoding-related midbrain activation also occurs independently of reward (Schott et al., 2004). The anatomical inference that this region is dopaminergic would be strengthened by physiological evidence if genetic polymorphisms related to dopamine metabolism affected its $\mathrm{AMRI}$ response.

The dopamine transporter (DAT1) plays a major role in presynaptic striatal dopamine uptake and thereby in control of extracellular dopamine levels in the striatum (Jaber et al., 1997). A variable number of tandem repeat (VNTR) polymorphism in the 
3' region of the DAT1 gene has been shown to affect DAT1 expression in vitro (Fuke et al., 2001; Mill et al., 2002) and in vivo (Heinz et al., 2000), with the 9-repeat allele showing lower DAT1 expression than the more frequent 10-repeat allele.

Catechol-O-methyl transferase (COMT) is important for dopamine degradation in the PFC, in which DAT1 expression is sparse (Karoum et al., 1994; Matsumoto et al., 2003). A singlenucleotide polymorphism leads to a valine to methionine substitution in the COMT protein (soluble form, position 108; membrane-bound form, position 158; Val108/158Met), resulting in threefold to fourfold lower enzymatic activity (Lotta et al., 1995). COMT Val108/158Met has been associated with frontal lobe function and risk for schizophrenia, with the Met allele conferring an advantage in several cognitive tasks (Egan et al., 2001). The Met allele has also been associated with superior performance in recall-based episodic memory tasks (de Frias et al., 2004).

Here we investigated the effects of DAT1 VNTR and COMT Val108/158Met on brain activity during episodic encoding using event-related fMRI. Given the anatomically dissociable roles of DAT1 and COMT in dopamine clearance, we hypothesized that DAT1 and COMT polymorphism might exert regionally specific influences on midbrain and prefrontal activations related to episodic encoding.

\section{Materials and Methods}

Participants. Fifty-one young (age range, 18-31 years), right-handed native speakers of German ( 35 female) were recruited for paid participation in the study. All were checked for MRI contraindications and gave written informed consent to participate. The study was approved by the local ethics committee. Subjects underwent routine clinical interview for history of neurological and psychiatric illnesses. Any present or past neurological or psychiatric disorders or the use of any centrally acting drugs was a contraindication for participation. All subjects had normal T1weighted anatomical MRIs.

Genotyping. Genomic DNA was extracted from venous blood samples of 49 participants, using the GenElute DNA extraction kit (Sigma, St. Louis, MO). Genotyping of DNA fragments containing the DAT1 VNTR (gene locus SLC6A3 on human chromosome 5p15) and the COMT Val108/158Met polymorphism (human chromosome 22q11; GenBank accession number Z26491) was performed using standard PCR methods (for detailed protocols, see supplemental data, available at www.jneurosci. org as supplemental material). Genotyping was also performed for a variety of additional polymorphisms (see below), to control for confounding effects of other polymorphisms and to reduce the risk of population stratification.

Paradigm. To investigate the correlates of successful memory formation, brain activity patterns during encoding of visually presented words were compared as a function of later remembering and forgetting (Wagner et al., 1998; Otten et al., 2002; Schott et al., 2004). The paradigm used in the present study has been shown to reliably elicit encoding-related activations of dopaminergic midbrain structures (Schott et al., 2004).

Participants studied words at two levels of processing (LOP) (deep vs shallow study processing). The experiment consisted of three sessions, each comprising three study phases with a deep study task and three study phases with a shallow study task. In the deep study task, participants were instructed to judge whether a word was pleasant or unpleasant and to respond with the index finger of one hand for pleasant and with the index finger of the other hand for unpleasant words. In the shallow study task, they were instructed to judge whether a word had exactly two syllables and to respond with one index finger for two syllables and with the other index finger for any other number of syllables. Response hands were counterbalanced across participants.

Twenty German words were presented per study list. Trials consisted of a central fixation cross for $500 \mathrm{~ms}$, a word presented for $1000 \mathrm{~ms}$, and an additional fixation cross for $1250 \mathrm{~ms}$. Study lists were followed by a distracter task (four moderately difficult arithmetic operations; subjects were asked to judge whether the result was correct and respond via button press). After the distracter task, a cue ("Please speak") prompted participants to a 90-s free-recall phase, in which they overtly recalled all studied words they could remember. Oral responses were recorded by a microphone placed at the bottom of the head coil and scored off-line. The session structure and trial timings are displayed in supplemental Figure 1 (available at www.jneurosci.org as supplemental material).

Functional MRI scanning. All MR images were acquired on a GE $1.5 \mathrm{~T}$ Signa Neurovascular system (General Electric Medical Systems, Milwaukee, WI) using a standard quadrature head coil. Three sessions of 544 echo-planar T2*-weighted MR images [repetition time (TR), $2.0 \mathrm{~s}$; echo time (TE), $35 \mathrm{~ms}$; 23 axial slices $(64 \times 64)$; voxel size, $3.13 \times 3.13 \times 6 \mathrm{~mm}$ ( $5 \mathrm{~mm}$ slice thickness, $1 \mathrm{~mm}$ gap)] were acquired in an interleaved manner (1-23 in steps of 2, 2-22 in steps of 2, from bottom to top). The first four volumes of each session were discarded.

Data processing and analysis. Data analysis was performed using Statistical Parametric Mapping (SPM2; Wellcome Department of Imaging Neuroscience, London, UK). Echo planar images were acquisition corrected, realigned, normalized (voxel size, $3 \times 3 \times 3 \mathrm{~mm}$ ) and smoothed (Gaussian kernel, $8 \times 8 \times 8 \mathrm{~mm}$ ). A high-pass filter of $128 \mathrm{~s}$ was applied to the data. Statistical analysis was performed in a two-stage mixedeffects model. In the first stage, neural activity was modeled by a delta function at stimulus onset. The blood oxygen level-dependent (BOLD) response was modeled by convolving these delta functions with a canonical hemodynamic response function (HRF). The resulting time courses were downsampled for each scan to form covariates in a general linear model. Covariates were modeled for the conditions of interest (deep hits, deep misses, shallow hits, and shallow misses), plus one for the speech events (overt responses in free recall), one for each of the six rigid-body movement parameters determined from realignment, one for the distracter task $(20 \mathrm{~s}$ epoch), and a single constant representing the mean over scans. Parameters for each covariate were estimated by an ordinary least-squares fit. Second-level random effects analyses were computed over the single subjects' contrasts. To specifically investigate mechanisms related to level of processing and successful encoding, respectively, difference contrasts ( $t$ statistics for deep vs shallow processing and remembered vs forgotten, respectively) were used for all second-level comparisons. To assess overall effects of LOP and subsequent memory performance, contrast images [LOP contrast: (deep hits + deep misses) vs (shallow hits + shallow misses); subsequent memory contrast: (deep hits + shallow hits) vs (deep misses + shallow misses)] were entered into one-sample $t$ tests. The significance level for the overall group analyses was set to 0.05 (whole-volume corrected), with an extent threshold of 10 adjacent voxels.

To investigate memory-related brain activity differences as a function of DAT1 genotype, carriers of the 9-repeat allele ( $n=17$; seven $9 / 9$, ten 9/10) were matched for age, sex, and years of education with 10-repeat homozygous subjects $(n=20)$ (for demographic data, see Table 1, left). To investigate between-group effects of COMT genotype, subsequent memory contrasts of $\mathrm{Val} / \mathrm{Val}(n=17)$ subjects and Met/Met subjects $(n=15)$ (Table 1 , right) were compared.

Because of our a priori hypothesis that DAT1 and COMT genotype would affect encoding-related activations in the midbrain, separate region of interest (ROI) analyses were performed for the left and right midbrain, respectively. The left and right substantia nigra was segmented manually from the normalized and averaged magnetization transfer (MT) (see below) image (see Fig. 2A), using the MRIcro image analysis software tool (http://www.sph.s.c.edu/comd/rorden/mricro.html). Using the Marsbar ROI analysis tool (Brett et al., 2002), two-sample $t$ tests (DAT1, 9-repeat carriers vs 10-homozygous subjects; COMT, Val/Val vs Met/Met) were computed over the mean midbrain contrast values in the subsequent memory contrast (subsequently remembered vs subsequently forgotten items). The significance threshold was set to $p=0.05$ (partial volume corrected).

To investigate the effects of DAT1 genotype on brain activations outside the midbrain, the subsequent memory contrasts were also submitted to voxelwise two-sample $t$ tests, with 9-repeat allele carriage as groupdefining factor. Similarly, voxelwise two-sample $t$ tests were used to com- 
Table. 1. Demographic information and behavioral data of DAT1 and COMT genotype groups

\begin{tabular}{|c|c|c|c|c|c|}
\hline & \multicolumn{2}{|l|}{ DAT1 VNTR } & \multicolumn{3}{|c|}{ COMT Val108/158Met } \\
\hline & $10 / 10$ & $9 / 9 ; 9 / 10$ & $\mathrm{Val} / \mathrm{Val}$ & $\mathrm{Val} / \mathrm{Met}$ & Met/Met \\
\hline$n$ & 20 & 17 & 17 & 17 & 15 \\
\hline Age & $23.1 \pm 3.0$ & $22.1 \pm 3.0$ & $23.1 \pm 3.1$ & $22.9 \pm 3.2$ & $22.3 \pm 2.7$ \\
\hline$M / F$ & $5 / 15$ & $4 / 13$ & $4 / 13$ & $5 / 14$ & $7 / 8$ \\
\hline Years of education & $15.9 \pm 2.9$ & $14.9 \pm 2.4$ & $15.8 \pm 3.0$ & $15.8 \pm 2.5$ & $14.8 \pm 1.9$ \\
\hline Deep hits & $0.36 \pm 0.104$ & $0.34 \pm 0.114$ & $0.36 \pm 0.121$ & $0.38 \pm 0.101$ & $0.34 \pm 0.088$ \\
\hline RT (deep hits) & $1409 \pm 262$ & $1500 \pm 160$ & $1425 \pm 221$ & $1494 \pm 271$ & $1423 \pm 227$ \\
\hline Deep misses & $0.62 \pm 0.102$ & $0.63 \pm 0.112$ & $0.61 \pm 0.115$ & $0.60 \pm 0.099$ & $0.64 \pm 0.085$ \\
\hline RT (deep misses) & $1431 \pm 250$ & $1504 \pm 182$ & $1434 \pm 230$ & $1514 \pm 258$ & $1437 \pm 227$ \\
\hline Shallow hits & $0.27 \pm 120$ & $0.26 \pm 0.118$ & $0.27 \pm 0.107$ & $0.29 \pm 0.122$ & $0.24 \pm 0.097$ \\
\hline RT (shallow hits) & $1386 \pm 271$ & $1372 \pm 176$ & $1327 \pm 253$ & $1434 \pm 222$ & $1382 \pm 245$ \\
\hline Shallow misses & $0.72 \pm 0.129$ & $0.73 \pm 0.118$ & $0.73 \pm 0.120$ & $0.70 \pm 0.124$ & $0.74 \pm 0.096$ \\
\hline RT (shallow misses) & $1364 \pm 269$ & $1376 \pm 172$ & $1322 \pm 235$ & $1429 \pm 240$ & $1364 \pm 230$ \\
\hline
\end{tabular}

Relative proportions of event types are given; differences to 1.0 result from unscorable items (e.g., ambiguous overt responses). $n$, Number of subjects; M/F, male/female; $\mathrm{RT}$, reaction time (in milliseconds).

pare brain activations outside the midbrain between COMT Val/Val homozygous subjects and COMT Met/Met homozygous subjects. The significance level for voxelwise between-group statistics was set to 0.005 (uncorrected), with a minimum of 10 adjacent voxels. Because we hypothesized a gene-dose effect of the COMT Val108/158Met polymorphism on memory-related brain activations, we also performed a regression analysis on the subsequent memory contrasts, with the number of Met alleles $(0,1$, or 2$)$ as independent variable.

To verify the reliability of the between-group effects for the DAT1 VNTR and the COMT Val108/158Met polymorphism, the SPM betas of the local maxima (recalled vs forgotten) were submitted to bootstrap resampling (Efron and Tibshirani, 1993). Specifically, the 95\% confidence intervals of the subsequent memory contrasts were estimated for both groups (DAT1, 10/10 vs 9-carriers and COMT, Val/Val vs Met/Met, respectively) using the percentile $t$ method (10,000 iterations for interval estimation; 200 iterations for variance estimation). Local maxima of activation with nonoverlapping 95\% confidence intervals in at least one study condition (deep or shallow) were considered reliable.

Functional connectivity analysis. To investigate the effects of the DAT1 VNTR and the COMT Val108/158Met polymorphism on the functional coupling between hippocampus and the prefrontal cortex, we used a modified version of the psychophysiological interactions (PPI) approach (Friston et al., 1997; Gitelman et al., 2003; Das et al., 2005) (for a similar use of PPI in genetic imaging, see Heinz et al., 2005). PPI analysis captures the functional coupling between different brain regions in relation to a psychological variable (in our study, successful vs unsuccessful encoding of study items). Because of its predominant role in the episodic encoding of verbal material, the left anterior hippocampus was chosen as source region for PPI analysis. A sphere with a radius of $6 \mathrm{~mm}$ was centered on the individual local maxima of activation within the left anterior hippocampus for each subject, and first eigen-variate time series from this sphere were extracted and deconvolved with the canonical HRF. The resulting time course was convolved with a psychological function $P$ of the time $t$ (in scans), which was set to +1 if $t$ was the onset of a remembered word, -1 if $t$ was the onset of a forgotten word, and 0 in all other cases. Reconvolution of the resulting function with the HRF yielded the vector $X$, which was entered as the first column of the design matrix of a new general linear model. $P$ itself was also convolved with the HRF to form a second covariate. The third covariate was the original BOLD eigenvariate. The six rigid-body movement parameters determined from realignment and a constant representing the mean over scans were included in the design matrix as covariates of no interest. Model estimation was performed as an ordinary least-squares fit. Voxelwise $t$ contrasts testing the interaction variable $X$ against baseline were computed for all individual subjects, and the resulting contrast images (hence referred to as PPI contrasts) were submitted to second-level random-effects analyses, as described above, using the same a priori statistical thresholds. Specifically, the functional coupling of the hippocampus with other brain regions was assessed using a voxelwise onesample $t$ test, and between-group differences were assessed by voxelwise two-sample $t$ tests of the single subjects' PPI contrasts.

MT scanning and image processing. Magnetization transfer images were obtained from five young, healthy male volunteers (age range, 18-27 years). Forty-eight slices (slice thickness, $3 \mathrm{~mm}$, no gap) of $256 \times 256$ voxels were acquired at a TR of $2600 \mathrm{~ms}$ and TE of $20 \mathrm{~ms}$. A magnetization transfer saturation pulse (off-resonance frequency of $1600 \mathrm{~Hz}$ ) was applied for $16 \mathrm{~ms}$. Images were normalized to the SPM2 protein density template (voxel size, $1 \times 1 \times 1 \mathrm{~mm}$ ), averaged to a mean image, which was then smoothed using a Gaussian kernel of $2.5 \mathrm{~mm}$.

Human brain material. Human brain material was obtained from the New Magdeburg Collection. Collection of human brain material was done in strict accordance with German laws and the rules outlined by the local ethics committee. Brains from three individuals (one female and two males, aged 52,54 , and 55 years) without any signs of neurological or psychiatric disorders were studied. The postmortem intervals were 21, 22 , and $24 \mathrm{~h}$, accordingly. One brain was flash frozen in liquid nitrogen for biochemical purposes, and the other two were processed for immunohistochemistry.

Immunohistochemical procedures. Brains were processed for histochemical analyses in a standard manner, including immersion fixation in $8 \%$ phosphate-buffered formaldehyde for 2 months, embedding in paraplast, and cutting with a microtome (whole brain sections, $20 \mu \mathrm{m}$ ). Every 50th section was histologically stained by combining the methods of Nissl and Heidenhain-Woelcke. To localize DAT1 immunoreactivity, we used a commercially available rabbit polyclonal antibody raised against a C-terminal fragment of human DAT1 (Santa Cruz Biotechnology, Santa Cruz, CA) The working dilution of the primary antiserum was 1:100 in PBS. The nickel-enhanced avidin-biotin technique was used as described previously (Bernstein et al., 1999). The specificity of the immunoreactivity was controlled by replacing the specific antibodies by buffer and Ig-free rabbit serum.

Protein preparation and Western blotting. Protein preparation and Western blotting were essentially performed as described previously (Seidenbecher et al., 2002), with minor modifications. Briefly, human brain tissue from the hippocampus (including dentate gyrus and CA1 and $\mathrm{CA} 3$ regions) and from the striatum was homogenized in TBS containing 1\% Triton X-100 and a protease inhibitor cocktail (Complete; Boehringer Mannheim, Mannheim, Germany). Homogenized protein probes were treated with a solubilizer containing SDS and mercaptoethanol. Proteins were separated by SDS-PAGE on $5-20 \%$ gels under fully reducing conditions, and transfer onto nitrocellulose was performed according to standard protocols. Western blots were immunodeveloped by overnight incubation with the primary antibody also used for histochemistry and processed using the enhanced chemiluminescence detection system (Amersham Biosciences, Arlington Heights, IL). 


\section{Results}

Genotyping

Blood samples for genotyping were available for 49 of our 51 participants. Within this group, 17 carriers of the DAT1 9-repeat allele were identified (seven 9/9, ten 9/10), which is within the expected range in a Caucasian population with allele frequencies of 0.72 for the 10 -repeat allele and 0.27 for the 9 -repeat allele (DoucetteStamm et al., 1995) $\left(\chi_{(2)}^{2}=4.232 ; p=\right.$ 0.121). The 9-repeat carriers were matched for age, gender, and years of education with 20 subjects homozygous for the 10-repeat allele. For the COMT Val108/158Met polymorphism, 17 subjects were Val homozygous, 15 subjects were Met homozygous, and the remaining 17 subjects were heterozygous. This distribution is within the expected range for a Caucasian population with approximately equal distribution of $\mathrm{Val}$ and Met alleles (DeMille et al., 2002) $\left(\chi_{(2)}^{2}=2.404 ; p=\right.$ $0.301)$. Detailed demographic data are displayed in Table 1. There was no significant difference in the distribution of COMT Val108/158Met genotypes between the DAT1 genotype groups $\left(\chi_{(2)}^{2}=2.817\right.$; $p=0.244)$.

To further control for effects of population stratification, genotyping was also performed for the dopamine receptor $\mathrm{D}_{2}$ TaqIA restriction fragment length polymorphism on human chromosome 11q23 and for the dopamine receptor $\mathrm{D}_{3}$ Ser9Gly polymorphism on human chromosome 3 q13 in all 49 subjects, as well as for the monoamine oxidase A promoter VNTR (human chromosome Xp11), the monoamine oxidase $\mathrm{B}$ intron 13 polymorphism (human chromosome Xp11), the brainderived neurotrophic factor Val66Met polymorphism (human chromosome 11p13), the serotonin transporter (SLC6A4) fragment length polymorphism (human chromosome 17q11), and the endothelial nitric oxide synthase Glu298Asp polymorphism (human chromosome 7q36) in 45 subjects (details available on request). Allelic distributions for these polymorphisms did not differ significantly for either DAT1 10-homozygous subjects and 9-repeat carriers or for COMT Val/Val and Met/Met subjects, thus making genetic inhomogeneity of the tested population unlikely.

\section{Behavioral results}

The average percentages of recalled items in the deep and shallow study condition were similar for DAT1 10-repeat homozygous subjects and 9-repeat carriers (Table 1, left) and for COMT Val/ Val, Val/Met, and Met/Met carriers (Table 1, right). Regardless of DAT1 or COMT genotype, LOP (deep vs shallow study task) had a strong effect on subsequent remembering of study items (interaction deep vs shallow processing $\times$ subsequent remembering vs forgetting, $F_{(1,46)}=83.6 ; p<0.001$, two-way ANOVA for repeated measures with COMT genotype as between-subjects factor). The DAT1 genotype did not affect either subsequent re- membering $\left(F_{(1,35)}=0.068 ; p=0.796\right)$ or the behavioral LOP effect (i.e., interaction between deep vs shallow processing and subsequent remembering; $F_{(1,35)}=0.059 ; p=0.810$, two-way ANOVA for repeated measures with DAT1 genotype as betweensubjects factor). There was also no effect of COMT genotype on memory performance $\left(F_{(1,46)}=0.836 ; p=0.440\right)$ or the behavioral LOP effect $\left(F_{(1,46)}=0.103 ; p=0.902\right)$.

Reaction times were significantly faster for shallowly studied items than for deeply studied items $\left(F_{(1,46)}=10.762 ; p=0.002\right)$, but there was no reaction time difference as a function of either subsequent recall or genotypes (all $p>0.167)$ (Table 1$)$.

\section{Functional MRI results}

Across the entire study cohort, characteristic brain activity patterns were observed for both LOP and subsequent memory contrasts. In the between-group comparisons, these brain activity patterns showed specific differences as a function of DAT1 and COMT genotypes, which will be described in detail below.

\section{LOP effects on brain activity}

Regardless of subsequent recall, areas activated more strongly in the deep than the shallow study condition were the bilateral me- 
dial frontal cortex [Brodmann area (BA) 6], the left inferior frontal gyrus (BA 45), the left amygdala, the left angular gyrus (BA 39), the left inferior and middle temporal gyrus (BA 21), and the bilateral superior temporal gyrus (BA 38) (Fig. 1A).

\section{Brain activity correlates of subsequent recall}

In our sample of 51 healthy adults, we observed that, during successful episodic memory formation, midbrain activations accompanied a fronto-parieto-occipital and limbic network activation, in line with previous observations in a smaller sample (Schott et al., 2004). Regardless of LOP, areas activated more strongly by subsequently recalled than forgotten words included the left dorsolateral prefrontal cortex (BA 45), the bilateral hippocampus and perirhinal cortices, bilateral caudate and nucleus accumbens, and parieto-occipital cortex, including primary and secondary visual areas (BA 17 and BA 18), as well as the ventral midbrain encompassing the medial substantia nigra and the ventral tegmental area (Fig. $1 B$ ). An ROI analysis of the segmented substantia nigra revealed a robust encoding-related activation of both the left and right midbrain (left, $T=5.26, p<0.0001$; right, $T=4.88, p<0.0001 ;$ small-volume corrections applied).

\section{Effects of DAT1 VNTR genotype on memory-related brain activity}

For the investigation of genotype effects on level of processing (deep vs shallow study), we conducted a between-group comparison of the LOP contrasts for DAT1 10-homozygous subjects and 9 -repeat carriers. Voxelwise $t$ test statistics revealed no reliable effects of DAT1 genotype on LOP for either 9-repeat carriers or 10 -repeat homozygous subjects.

To investigate the effects of DAT1 genotype on brain activation patterns related to successful memory formation, we compared fMRI contrasts of 10-repeat homozygous subjects of the DAT1 VNTR with those of subjects carrying at least one 9-repeat allele. Compared with 10-repeat homozygous subjects, 9-repeat carriers showed relatively higher activation of the dopaminergic midbrain. The ROI analysis revealed that, compared with 10/10 subjects, 9-repeat carriers exhibited higher activation of the right midbrain/substantia nigra as a function of subsequent recall (subsequently recalled vs forgotten words) (Fig. $2 \mathrm{~B}$, left). This effect was significant after partial volume correction for multiple comparisons $(T=2.53 ; p=0.016)$. The voxelwise $t$ statistic revealed that the difference in midbrain activation was most prominent over the anterior medial midbrain (Fig. 3A). Additionally, 9-repeat carriers showed relatively higher memoryrelated activation of the anterior cingulate (Fig. $3 B$, left) and parts of the basal forebrain, including the ventral striatum, the inferior medial prefrontal cortex, and the subgenual portion of the anterior cingulate (Fig. $3 B$, right), regions that can exert a net excitatory effect on midbrain dopaminergic neurons (Lisman and Otmakhova, 2001). These findings were reliable, as estimated by bootstrap resampling, with nonoverlapping 95\% confidence intervals of the SPM betas in at least one study condition. Table 2 summarizes the clusters of activation with significantly higher activations in carriers of the 9-repeat allele.

\section{Effects of COMT Val108/158Met genotype on memory- related brain activity}

To assess the effects of COMT Val108/158Met genotype on the level of study processing, voxelwise $t$ statistics were computed for the LOP contrasts for Val/Val and Met/Met subjects. Compared with Met homozygous subjects, Val homozygous subjects showed increased activation of the right prefrontal cortex and of
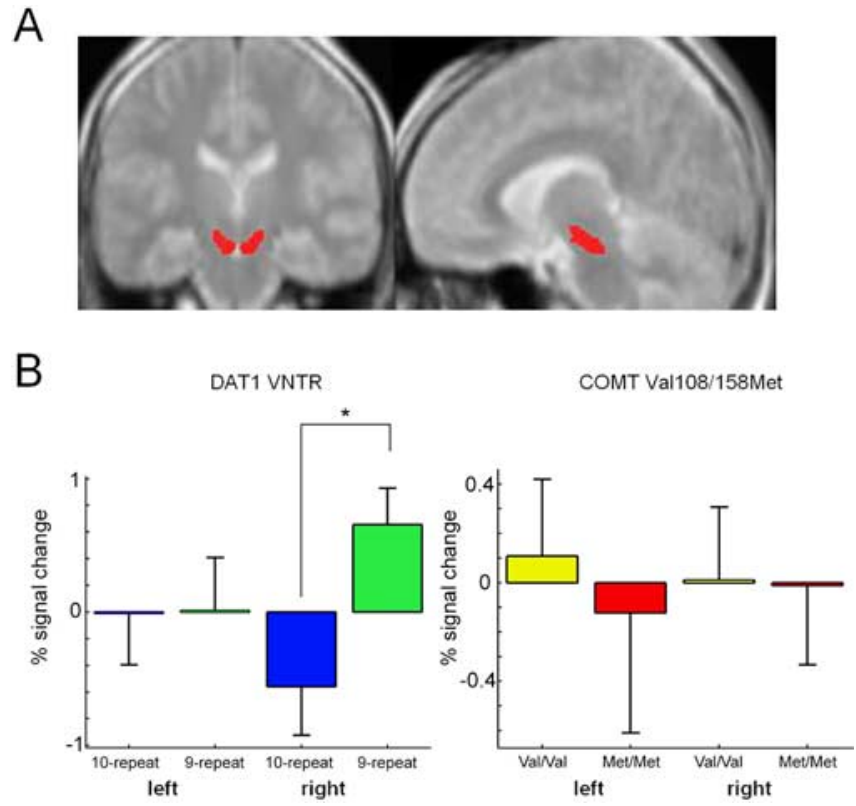

Figure 2. Effects of DAT1 and COMT genotypes on memory-related midbrain activations. $\boldsymbol{A}$, ROls encompassing the left and right substantia nigra and the ventral tegmental area. The ROls were segmented manually from an average of five normalized MT images. $\boldsymbol{B}$, Results of the ROI analyses in the left and right midbrain for the DAT1 VNTR (left) and the COMT Val108/158Met polymorphism (right). The 9-repeat carriers of the DAT1 VNTR showed significantly higher activation of the right midbrain during successful episodic memory formation. ${ }^{*} p<0.05$, partial volume corrected.

the left fusiform gyrus during deep versus shallow processing Met homozygous subjects showed increased activation of the posterior cingulate relative to $\mathrm{Val} / \mathrm{Val}$ carriers during deep versus shallow processing. Supplemental Figure 2 (available at www. jneurosci.org as supplemental material) displays typical activation differences for the LOP contrast.

To investigate effects of the COMT Val108/158Met polymorphism on brain activity during successful episodic encoding, the subsequent memory contrasts of COMT Val homozygous and Met homozygous participants were compared. In contrast to the DAT1 VNTR, the COMT Val108/158Met genotype was not associated with encoding-related brain activity differences in the midbrain/substantia nigra. The ROI analyses revealed no significant activation differences in either left or right midbrain/substantia nigra between COMT108/158Val and Met homozygous subjects as a function of subsequent recall performance (Fig. $2 B$, right). However, cortical activation patterns showed robust differences between the two groups (Fig. 4). Compared with Met/ Met subjects, Val/Val subjects showed increased memory-related activations of the right prefrontal cortex (BA 8 and BA 9) (Fig. $4 A$ ) and the left fusiform gyrus (BA 19), comparable with the LOP-related activation difference in these regions and similar to right prefrontal activation differences observed in a working memory task (Egan et al., 2001). COMT Val homozygous subjects also showed relatively higher activations in portions of the medial occipital cortex that extended into the primary visual cortex (cuneus; BA 17 and BA 18) (Fig. 4B). The prefrontal and medial occipital activation differences were reliable, as estimated by bootstrap resampling (see above). In the medial occipital cortex, however, 95\% confidence intervals of the mean activations showed considerable overlap in the deep study condition, suggesting that the latter difference might primarily be confined to shallow (i.e., perceptual) encoding. A complete list of memory- 
related activation differences between $\mathrm{Val} /$ Val and Met/Met subjects is displayed in Table 3.

To exclude a possible confound attributable to the unequal gender distribution in the $\mathrm{Val} / \mathrm{Val}$ and Met/Met groups, subgroups were selected for age match (eight females and four males from each group; average age, 22.7 years in the $\mathrm{Val} / \mathrm{Val}$ and 22.4 years in the Met/Met subgroup), and the SPM contrasts (hits-misses) of these subgroups were submitted to a voxelwise two-sample $t$ test $(p<0.005$, uncorrected; minimum of 10 adjacent voxels). Like in the entire cohort, the subgroup of $\mathrm{Val} / \mathrm{Val}$ subjects showed higher activations of the right prefrontal $([x, y, z]=[33,18,21])$ and medial occipital $([x, y, z]=[9,-87$, 12]) cortices compared with the Met/Met subgroup.

Voxelwise regression analysis revealed that COMT Val108/158Met heterozygous subjects $(n=17)$ showed intermediate activation levels in right prefrontal cortex $([x, y, z]=[30,18,21] ; T=3.69 ; p<$ $0.001)$ and in the bilateral medial occipital cortex $([x, y, z]=[9,-87,12] ; T=4.94$; $p<0.001)$.

Effects of DAT1 and COMT genotypes on functional coupling of the hippocampus and prefrontal cortex

As reported in previous studies, genetic polymorphisms can affect not only differences in overall brain activation but also the functional connectivity between different brain regions (Heinz et al., 2005; Pezawas et al., 2005). Here we used a psychophysiological interaction approach (Friston et al., 1997; Gitelman et al., 2003) to investigate a possible influence of DAT1 and COMT polymorphisms on memory-related functional coupling between the hippocampus and the prefrontal cortex. Because of our a priori knowledge of the involvement of prefrontal cortex in successful verbal episodic memory encoding (Fig. $1 B$ ) and guided by the results of our $t$ statistic-based comparisons of genotype groups (Figs. 3, 4), we restricted our PPI analysis to the functional coupling of the left hippocampus and structures within the frontal lobes.

Regardless of genotype, successful encoding of words was associated with increased functional coupling between the left hippocampus and the bilateral dorsal prefrontal cortex and the left orbitofrontal cortex (data not shown). A voxelwise two-sample $t$ test comparison of the PPI contrasts of DAT1 9-repeat carriers and 10-homozygous subjects revealed that the DAT1 VNTR affected coupling of the left hippocampus with inferior portions of the right and left orbitofrontal cortex and the left basal forebrain $([x, y, z]=[-15,0,-9] ; T=3.31)$, with 9-repeat carriers showing relatively higher functional coupling of the hippocampus with these brain regions.

$t$ test-based comparison of the PPI contrasts for COMT Val/ Val and Met/Met subjects showed that COMT Val108/158Met polymorphism was strongly associated with functional coupling of the hippocampus and the bilateral prefrontal cortex during successful encoding (Fig. 5). Notably, when compared with Val/ Val individuals, Met/Met homozygous subjects showed relatively
Table 2. Regions showing activation differences as a function of DAT1 VNTR genotype

\begin{tabular}{lrrrr}
\hline & $x$ & $y$ & $z$ & $T$ \\
\hline 9 repeat $>10$ repeat & & & & \\
$\quad$ Right anterior cingulate, BA 32* & 12 & 42 & 6 & 3.60 \\
Right basal forebrain* & 3 & 15 & -9 & 4.61 \\
Left anterior cingulate, BA 32* & -6 & 27 & -6 & 3.12 \\
& -12 & 21 & 24 & 3.25 \\
Left subcallosal gyrus, BA 11* & -12 & 21 & -12 & 3.24 \\
Right midbrain, substania nigra* & 9 & -15 & -15 & 3.52 \\
Left caudate* & -18 & -27 & 21 & 3.45 \\
Left insula, BA 13* $_{\text {Left postcentral gyrus, BA 2 }}$ & -27 & -30 & 27 & 4.37 \\
Right parahippocampal gyrus, BA 36 & -36 & -33 & 30 & 3.02 \\
Left parahippocampal gyrus, BA 19 & 18 & -42 & -18 & 3.35 \\
Left fusiform gyrus, BA 37 & -21 & -54 & -12 & 3.32 \\
& -33 & -51 & -18 & 3.63 \\
Left parietal lobe* & -33 & -57 & -12 & 3.33 \\
& -21 & -54 & 18 & 3.28 \\
Right parietal lobe & -27 & -54 & 9 & 3.33 \\
Right cerebellum* & 30 & -54 & 18 & 3.48 \\
& 27 & -51 & -42 & 3.75 \\
10 repeat $>$ 9 repeat & 30 & -54 & -30 & 3.13 \\
(19 & & & &
\end{tabular}

10 repeat $>9$ repeat

No voxels survive extent threshold, $k=10$

Local maxima are given in MNI coordinates. Regions marked with an asterisk show reliable activation differences, as estimated by bootstrap resampling of the SPM betas.

increased functional coupling of the hippocampus and the left and right PFC, albeit exhibiting relatively lower encoding-related activity in the right PFC. This association of the Met allele with higher functional coupling between the hippocampus and the PFC remained significant in the comparison of the gendermatched subgroups $([x, y, z]=[-39,39,9] ; T=3.85)$. Regression analysis showed that heterozygous subjects showed an inter- 


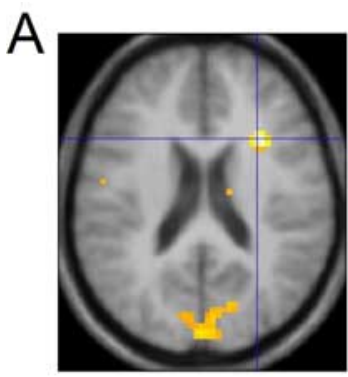

$[x, y, z]=[30,18,21]$ $\mathrm{T}=5.48$

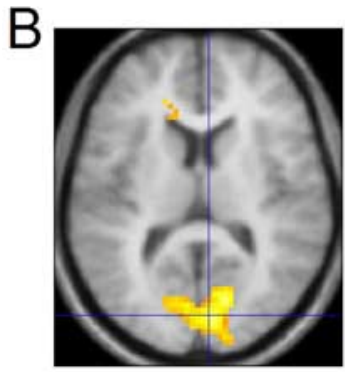

$[x, y, z]=[6,-84,12]$ $\mathrm{T}=4.52$

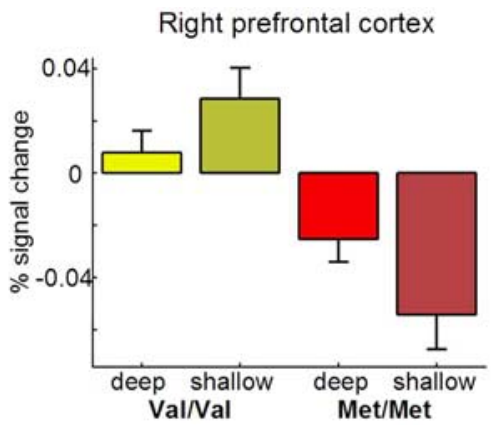

Cuneus

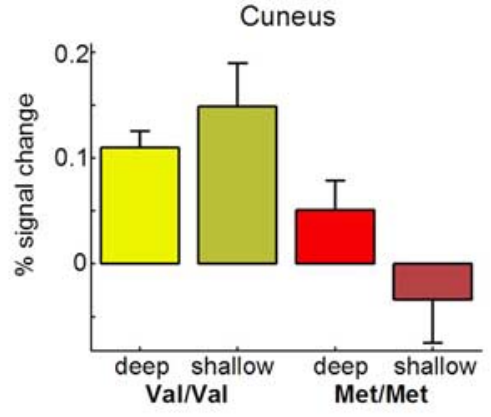

Figure 4. Effects of COMT Val108/158Met polymorphism on memory-related brain activity. Compared with Met homozygous subjects, Val homozygous subjects show increased memoryrelated activations of the right prefrontal cortex $(\boldsymbol{A})$ and of the cuneus, extending into the primary visual cortex $(\boldsymbol{B})$. Activations above a threshold of $T>2.72(p<0.005$, uncorrected; extent threshold, $k=10$ voxels) are displayed. Bar plots display the same information as in Figure 3. $[x, y, z]$, Voxel coordinates in MNI reference space.

Table 3. Regions showing activation differences as a function of COMT Val108/ 158Met genotype

\begin{tabular}{lrrrr}
\hline & $x$ & $y$ & $z$ & $T$ \\
\hline Val/Val > Met/Met & & & & \\
Right prefrontal cortex, BA 6, BA 47* & 30 & 18 & 21 & 5.48 \\
Right inferior frontal gyrus, BA 47* & 45 & 30 & -15 & 3.14 \\
Left anterior cingulate, BA 32 & -18 & 36 & 12 & 3.45 \\
Left cingulate gyrus, BA 32 & -3 & 21 & 30 & 3.68 \\
Right superior frontal gyrus, BA 6 & 9 & 12 & 72 & 3.38 \\
Left superior frontal gyrus, BA 6 & 0 & 6 & 69 & 3.75 \\
Left frontal lobe & -27 & -12 & 36 & 3.57 \\
Right caudate* & 15 & -15 & 27 & 3.43 \\
Left caudate* & -12 & 27 & 15 & 3.61 \\
Left precentral gyrus, BA 4, BA 6* & -42 & -18 & 42 & 3.57 \\
& -60 & -3 & 27 & 3.28 \\
Left fusiform gyrus, BA 19* & -54 & -6 & 21 & 2.91 \\
Right cerebellum & -30 & -66 & -27 & 4.90 \\
Right cuneus, BA 17, BA 18* & 30 & -69 & -30 & 3.41 \\
& 15 & -75 & 15 & 4.79 \\
& 6 & -84 & 12 & 4.52 \\
& -3 & -90 & 6 & 3.78
\end{tabular}

Met/Met $>\mathrm{Val} / \mathrm{Val}$

No voxels survive extent threshold, $k=10$

Local maxima are given in MNI coordinates. Regions marked with an asterisk show reliable activation differences, as estimated by bootstrap resampling of the SPM betas.

mediate level of fronto-hippocampal coupling during successful encoding $([x, y, z]=[-39,39,9] ; T=4.67)$.

\section{Dopamine transporter expression in the}

\section{human hippocampus}

Although expression of the human dopamine transporter has been reported at high levels in the striatum and nucleus accum- bens and at low levels in the prefrontal cortex (Ciliax et al., 1999), no data have, thus far, been available on hippocampal dopamine transporter expression in humans. To better understand the selective distribution of the encoding-related hemodynamic effects of the DAT1 polymorphisms, we therefore investigated the expression of DAT1 in the human hippocampus using immunohistochemistry and Western blotting. Histochemical results showed that DAT1 immunoreactivity was widely, but unevenly, distributed in the human CNS. In the hippocampus, a fine network of DAT1-immunoreactive fibers was found in the CA3 and CA4 regions (Fig. 6A). The dentate gyrus showed a more punctuate localization of dopamine transporter immunoreactivity. A small number of neurons located in the subiculum were surrounded by DAT1-immunoreactive material (Fig. 6B). No detectable levels of DAT1 immunoreactivity, however, were observed in the CA1 region of the hippocampus. To verify the validity of these findings, DAT1 immunoreactivity was also assessed in the putamen and caudate nucleus, revealing dense patterns of DAT1immunoreactive fibers (supplemental Fig. 3, available at www. jneurosci.org as supplemental material). DAT1 immunoreactivity was also observed within the substantia nigra, replicating previous observations (Ciliax et al., 1999). Control sections with antibody-free rabbit serum replacing the primary antibody lacked any immunostaining (supplemental Fig. 3, available at www.jneurosci.org as supplemental material). Western blotting using the same primary antibody as in the histochemical investigations revealed the expression of a DAT1-immunoreactive protein of $\sim 85 \mathrm{kDa}$ in the human striatum and, to a lesser extent, also in the human hippocampus (Fig. 6C).

\section{Discussion}

Our findings that genetic variations in the dopamine clearance pathways affect encoding-related activation patterns in midbrain and prefrontal cortex provide support for our hypothesis that dopaminergic transmission is involved in episodic memory encoding.

The DAT1 VNTR affected memory-related activation of the dopaminergic midbrain itself, whereas no comparable effect in the midbrain was observed for the COMT Val108/158Met polymorphism. However, cortical activation patterns and frontohippocampal coupling varied as a function of COMT genotype. Although our approach did not allow us to directly measure dopamine release, our findings are compatible with animal research and human postmortem studies, which show that DAT1 is abundantly expressed in midbrain and striatum but sparsely in the prefrontal cortex (Sesack et al., 1998), whereas COMT is particularly involved in prefrontal dopamine degradation.

\section{DAT1 VNTR genotype and memory-related} midbrain activation

The 9-repeat allele of the DAT1 VNTR has been shown previously to be associated with reduced DAT1 gene expression in vitro (Fuke et al., 2001; Mill et al., 2002). In humans, striatal dopamine transporter availability, as measured by single photon emission tomography, was reduced in 9-repeat carriers (Heinz et al., 2000), although the evidence is not entirely conclusive, and one study showed a reverse pattern (Jacobsen et al., 2000).

In our study, the 9-repeat allele was associated with a relatively increased midbrain response during episodic encoding. This observation is somewhat counterintuitive under the assumption that DAT1 9-repeat carriers have a lower striatal dopamine transporter density and presumably higher extracellular dopamine 
levels. Studies in dopamine transporterdeficient mice, however, have shown that lack of dopamine transporters leads to extensive adaptive changes in the dopaminergic system (Jones et al., 1998), including reduced tyrosine hydroxylase levels, higher expression of autoinhibitory presynaptic $\mathrm{D}_{2}$ receptors, and decreased activity-dependent dopamine release. We hypothesize that, in DAT1 9-repeat carriers, lower DAT1 levels might lead to similar adaptation processes. The relatively higher midbrain activation in DAT1 9-repeat carriers versus 10-homozygous subjects was specific for the successful encoding of items, a short, phasic process. We speculate that the average levels of activity-dependent dopamine release might be lower in 9-repeat carriers relative to 10-homozygous individuals. During hippocampal processing of novel items, stimulation of midbrain dopaminergic neurons by cell populations in the basal forebrain or nucleus accumbens, areas that showed higher memory-related activation in 9-repeat carriers, might compensate for this lower average midbrain activity by increased driving of midbrain dopaminergic neurons, thus occasionally leading to similar levels of midbrain activity as in 10-homozygous subjects (see our model in supplemental Fig. 4, available at www.jneurosci.org as supplemental material). If dopamine release indeed promoted hippocampus-dependent encoding processes (Lisman and Grace, 2005), events accompanied by this increased dopaminergic activity might be particularly likely to be encoded into hippocampusdependent memory. Compatible with this possibility, the hippocampus showed relatively stronger functional coupling with basal forebrain regions in 9-repeat carriers.

DAT1 availability has been positively previously correlated with memory performance (Mozley et al., 2001). In our study, no behavioral effect of DAT1 genotype was observed in our study. It is possible that, in our young, healthy study population, differences in DAT1 availability were primarily compensated on the level of midbrain, maybe by relatively increased stimulation of midbrain neurons by basal forebrain regions. Compatible with our hypothesis of compensation on the level of midbrain and its afferences, DAT1 genotype did not affect memory-related hippocampal activation, despite the observed DAT1 expression in the human hippocampus (Fig. 6). An effect of the DAT1 VNTR on memory performance might, however, become apparent when testing populations with alterations in prefrontal and hippocampal memory systems, such as elderly subjects or patients with neurological or psychiatric disorders.

Alternatively, the DAT1 VNTR might modulate memoryrelated midbrain activation by affecting firing of GABAergic neurons of the substantia nigra, pars reticulata. In our view, however, the exclusively presynaptic distribution of DAT1 (Piccini, 2003) make this possibility less likely.

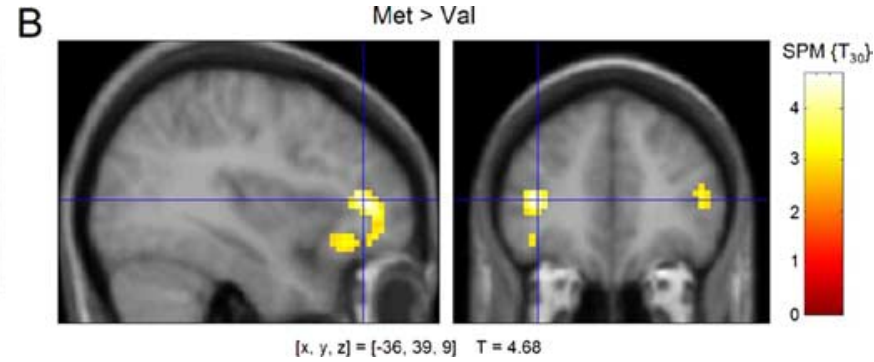

$[x, y, z]=[-36,39,9] \quad T=4.68$

Figure 5. Effects of COMT Val108/158Met polymorphism on functional coupling of the prefrontal cortex and the hippocampus up to 32 voxels. $\boldsymbol{B}$, Compared with Val homozygous subjects, Met/Met carriers showed significantly stronger functional coupling
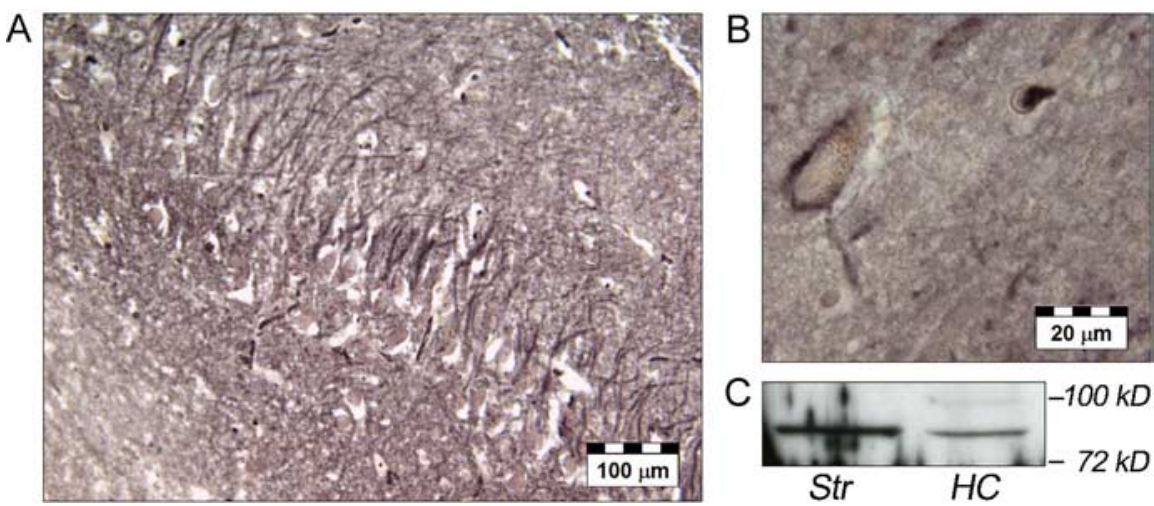

Figure 6. Expression of DAT1 in the human hippocampus. $\boldsymbol{A}$, In the CA3 region, a fine network of DAT1-immunoreactive fibers could be observed. $\boldsymbol{B}$, In the subiculum, a small subset of DAT1-immunoreactive neurons stood out. $\boldsymbol{C}$, Western blotting revealed a DAT1-immunoreactive band at $\sim 85 \mathrm{kDa}$ in both the human striatum and hippocampus. HC, Hippocampus; Str, striatum.

\section{Effects of COMT Val108/158Met genotype on memory- related brain activity}

The quantitative contribution of COMT to dopamine degradation is under debate, with several studies suggesting that its role is secondary to that of dopamine reuptake and oxidation by monoamine oxidase A and B (Budygin et al., 1999; Huotari et al., 2002, 2004). These studies have focused on dopamine metabolism in the striatum in which DAT1 expression is abundant. However, in the prefrontal cortex, in which DAT1 is expressed sparsely, COMT appears to play a more important role in the clearance of released dopamine (Karoum et al., 1994; Matsumoto et al., 2003; Tunbridge et al., 2004). Moreover, the COMT Val108/158Met polymorphism, which leads to lower enzyme activity in Met carriers, affects midbrain tyrosine hydroxylase levels and dopamine synthesis (Akil et al., 2003) and modulates interactions between the dopaminergic midbrain and the prefrontal cortex (MeyerLindenberg et al., 2005). A role for COMT in prefrontal dopamine degradation is compatible with our observation that the COMT Val108/158Met polymorphism, which leads to lower enzyme activity in Met homozygous subjects, did not affect memory-related midbrain activations in our study but modulated prefrontal activation patterns. It should be noted that, in our study, midbrain activity was measured in an event-related manner, possibly representing a correlate of brief, phasic fluctuations in dopamine release, whereas Meyer-Lindenberg et al. (2005) observed a more tonic effect of COMT Val108/158Met on midbrain dopamine metabolism. Such a tonic effect is not ex- 
cluded by the lack of influence of COMT genotype on phasic midbrain activations.

Relatively higher prefrontal activity in COMT108/158 Val/Val subjects has been suggested to be a correlate of decreased prefrontal processing efficiency in cognitive tasks such as working memory (Egan et al., 2001). Here we observed increased right prefrontal activation in Val/Val subjects for both higher level of processing (supplemental Fig. 2, available at www.jneurosci.org as supplemental material) and more successful encoding (Fig. 4), suggesting that higher prefrontal activations in $\mathrm{Val} / \mathrm{Val}$ subjects might be a phenomenon common to several cognitive tasks. It might indicate a compensatory mechanism for relatively lower prefrontal dopamine. In line with this notion, Parkinson's patients show relatively higher right prefrontal activation in working memory tasks compared with controls, and this activation decreases after the patients have received L-dopa (Cools et al., 2002; Mattay et al., 2002). Unlike in a previous study (de Frias et al., 2004) in a larger population, COMT Val108/158Met did not affect memory performance in our study, possibly attributable to our relatively small sample size or because our young, healthy subjects could recruit additional cognitive resources to compensate for relative dopamine deficiency. Notably, whereas overall activity in right PFC was stronger in Val homozygous subjects, Met homozygous subjects showed stronger functional coupling of the PFC and the hippocampus during successful encoding. This supports the possibility that COMT Val108/158Met might affect prefrontal processing efficiency and thereby facilitate the transfer of information between the PFC and the hippocampus. Such relatively stronger functional coupling between the PFC and the hippocampus might be a neural basis for higher episodic memory performance in Met/Met subjects observed previously (de Frias et al., 2004).

\section{Activations in early visual areas}

Compared with subsequently forgotten items, subsequently recalled items elicited higher activity in early visual areas (Fig. $1 B$ ). Although a role for the cuneus in memory processes has been reported (Tulving et al., 1999; Addis et al., 2004), the observation that activations in these regions were affected by COMT genotype was surprising. Val/Val subjects exhibited higher medial occipital activations (Fig. $4 B$ ) than Met/Met subjects, particularly in the shallow study condition, when subjects were likely to rely more on perceptual features of the study item. Because primary visual cortex has relatively few dopaminergic afferences (Berger et al., 1991), a local dopaminergic effect is unlikely. Therefore, the effect might be indirectly related to altered prefrontal processing (Barcelo et al., 2000; Chudasama and Robbins, 2004), or, alternatively, it might be related to noradrenaline, which is also metabolized by COMT. There is abundant noradrenergic input to early visual areas (Morrison et al., 1982), which has been shown to increase visuocortical signal-to-noise ratio ( $\mathrm{Si}$ ciliano et al., 1999). Higher visuocortical activity in $\mathrm{Val} / \mathrm{Val}$ subjects might thus reflect a compensatory mechanism for a lower signal-to-noise ratio attributable to faster noradrenaline degradation.

\section{Activation differences in absence of performance differences}

Similar to previous fMRI studies of genetic polymorphisms (Bookheimer et al., 2000; Egan et al., 2001; Hariri et al., 2002), between-group differences in brain activity were not associated with performance differences between genotype groups, suggesting the presence of compensatory mechanisms in the brain that allow healthy humans to achieve similar levels of performance despite interindividual differences in metabolic processes. Studying these mechanisms might help to further elucidate the interaction of normal genetic variations with physiological (e.g., aging) or pathological (e.g., neuropsychiatric disorders) alterations of brain function, thereby broadening our understanding of the pathogenesis of neuropsychiatric diseases with polygenic etiology.

\section{Conclusions}

Our data provide evidence for a functional role of dopaminergic midbrain regions in episodic memory encoding. Additionally, the observation that a genetic variation leading to differences in dopamine transporter expression levels appears to influence memory-related midbrain activity, whereas a genetic polymorphism that affects COMT activity appears primarily affect prefrontal brain processes in episodic memory formation is in line with previous evidence for regionally specific roles of DAT1 and COMT in dopamine clearance.

\section{References}

Aalto S, Bruck A, Laine M, Nagren K, Rinne JO (2005) Frontal and temporal dopamine release during working memory and attention tasks in healthy humans: a positron emission tomography study using the high-affinity dopamine $\mathrm{D}_{2}$ receptor ligand $\left[{ }^{11} \mathrm{C}\right]$ FLB 457. J Neurosci 25:2471-2477.

Addis DR, McIntosh AR, Moscovitch M, Crawley AP, McAndrews MP (2004) Characterizing spatial and temporal features of autobiographical memory retrieval networks: a partial least squares approach. NeuroImage 23:1460-1471.

Akil M, Kolachana BS, Rothmond DA, Hyde TM, Weinberger DR, Kleinman JE (2003) Catechol-O-methyltransferase genotype and dopamine regulation in the human brain. J Neurosci 23:2008-2013.

Bach ME, Barad M, Son H, Zhuo M, Lu YF, Shih R, Mansuy I, Hawkins RD, Kandel ER (1999) Age-related defects in spatial memory are correlated with defects in the late phase of hippocampal long-term potentiation in vitro and are attenuated by drugs that enhance the cAMP signaling pathway. Proc Natl Acad Sci USA 96:5280-5285.

Barcelo F, Suwazono S, Knight RT (2000) Prefrontal modulation of visual processing in humans. Nat Neurosci 3:399-403.

Berger B, Gaspar P, Verney C (1991) Dopaminergic innervation of the cerebral cortex: unexpected differences between rodents and primates. Trends Neurosci 14:21-27.

Bernstein HG, Baumann B, Danos P, Diekmann S, Bogerts B, Gundelfinger ED, Braunewell KH (1999) Regional and cellular distribution of neural visinin-like protein immunoreactivities (VILIP-1 and VILIP-3) in human brain. J Neurocytol 28:655-662.

Bookheimer SY, Strojwas MH, Cohen MS, Saunders AM, Pericak-Vance MA, Mazziotta JC, Small GW (2000) Patterns of brain activation in people at risk for Alzheimer's disease. N Engl J Med 343:450-456.

Brett M, Anton J-L, Valabregue R, Poline J-B (2002) Region of interest analysis using an SPM toolbox (CD-ROM). NeuroImage 16.

Buckner RL, Logan J, Donaldson DI, Wheeler ME (2000) Cognitive neuroscience of episodic memory encoding. Acta Psychol (Amst) 105:127-139.

Budygin EA, Gainetdinov RR, Kilpatrick MR, Rayevsky KS, Mannisto PT, Wightman RM (1999) Effect of tolcapone, a catechol-Omethyltransferase inhibitor, on striatal dopaminergic transmission during blockade of dopamine uptake. Eur J Pharmacol 370:125-131.

Chudasama Y, Robbins TW (2004) Dopaminergic modulation of visual attention and working memory in the rodent prefrontal cortex. Neuropsychopharmacology 29:1628-1636.

Ciliax BJ, Drash GW, Staley JK, Haber S, Mobley CJ, Miller GW, Mufson EJ, Mash DC, Levey AI (1999) Immunocytochemical localization of the dopamine transporter in human brain. J Comp Neurol 409:38-56.

Cools R, Stefanova E, Barker RA, Robbins TW, Owen AM (2002) Dopaminergic modulation of high-level cognition in Parkinson's disease: the role of the prefrontal cortex revealed by PET. Brain 125:584-594.

Das P, Kemp AH, Liddell BJ, Brown KJ, Olivieri G, Peduto A, Gordon E, Williams LM (2005) Pathways for fear perception: modulation of amygdala activity by thalamo-cortical systems. NeuroImage 26:141-148. 
de Frias CM, Annerbrink K, Westberg L, Eriksson E, Adolfsson R, Nilsson LG (2004) COMT gene polymorphism is associated with declarative memory in adulthood and old age. Behav Genet 34:533-539.

DeMille MM, Kidd JR, Ruggeri V, Palmatier MA, Goldman D, Odunsi A, Okonofua F, Grigorenko E, Schulz LO, Bonne-Tamir B, Lu RB, Parnas J, Pakstis AJ, Kidd KK (2002) Population variation in linkage disequilibrium across the COMT gene considering promoter region and coding region variation. Hum Genet 111:521-537.

Doucette-Stamm LA, Blakely DJ, Tian J, Mockus S, Mao JI (1995) Population genetic study of the human dopamine transporter gene (DAT1). Genet Epidemiol 12:303-308.

Eckert T, Sailer M, Kaufmann J, Schrader C, Peschel T, Bodammer N, Heinze H, Schoenfeld M (2004) Differentiation of idiopathic Parkinson's disease, multiple system atrophy, progressive supranuclear palsy, and healthy controls using magnetization transfer imaging. NeuroImage 21:229-235.

Efron B, Tibshirani R (1993) An introduction to the bootstrap. New York: Chapman and Hall.

Egan MF, Goldberg TE, Kolachana BS, Callicott JH, Mazzanti CM, Straub RE, Goldman D, Weinberger DR (2001) Effect of COMT Val108/158 Met genotype on frontal lobe function and risk for schizophrenia. Proc Natl Acad Sci USA 98:6917-6922.

Egan MF, Kojima M, Callicott JH, Goldberg TE, Kolachana BS, Bertolino A, Zaitsev E, Gold B, Goldman D, Dean M, Lu B, Weinberger DR (2003) The BDNF val66met polymorphism affects activity-dependent secretion of BDNF and human memory and hippocampal function. Cell 112:257-269.

Friston KJ, Buechel C, Fink GR, Morris J, Rolls E, Dolan RJ (1997) Psychophysiological and modulatory interactions in neuroimaging. NeuroImage 6:218-229.

Fuke S, Suo S, Takahashi N, Koike H, Sasagawa N, Ishiura S (2001) The VNTR polymorphism of the human dopamine transporter (DAT1) gene affects gene expression. Pharmacogenomics J 1:152-156.

Gabrieli JD, Brewer JB, Desmond JE, Glover GH (1997) Separate neural bases of two fundamental memory processes in the human medial temporal lobe. Science 276:264-266.

Gasbarri A, Sulli A, Innocenzi R, Pacitti C, Brioni JD (1996) Spatial memory impairment induced by lesion of the mesohippocampal dopaminergic system in the rat. Neuroscience 74:1037-1044.

Gitelman DR, Penny WD, Ashburner J, Friston KJ (2003) Modeling regional and psychophysiologic interactions in fMRI: the importance of hemodynamic deconvolution. NeuroImage 19:200-207.

Hariri AR, Mattay VS, Tessitore A, Kolachana B, Fera F, Goldman D, Egan MF, Weinberger DR (2002) Serotonin transporter genetic variation and the response of the human amygdala. Science 297:400-403.

Heinz A, Goldman D, Jones DW, Palmour R, Hommer D, Gorey JG, Lee KS, Linnoila M, Weinberger DR (2000) Genotype influences in vivo dopamine transporter availability in human striatum. Neuropsychopharmacology 22:133-139.

Heinz A, Braus DF, Smolka MN, Wrase J, Puls I, Hermann D, Klein S, Grusser SM, Flor H, Schumann G, Mann K, Büchel C (2005) Amygdalaprefrontal coupling depends on a genetic variation of the serotonin transporter. Nat Neurosci 8:20-21.

Huotari M, Santha M, Lucas LR, Karayiorgou M, Gogos JA, Mannisto PT (2002) Effect of dopamine uptake inhibition on brain catecholamine levels and locomotion in catechol-O-methyltransferase-disrupted mice. J Pharmacol Exp Ther 303:1309-1316.

Huotari M, Garcia-Horsman JA, Karayiorgou M, Gogos JA, Mannisto PT (2004) D-Amphetamine responses in catechol-O-methyltransferase (COMT) disrupted mice. Psychopharmacology 172:1-10.

Jaber M, Jones S, Giros B, Caron MG (1997) The dopamine transporter: a crucial component regulating dopamine transmission. Mov Disord 12:629-633.

Jacobsen LK, Staley JK, Zoghbi SS, Seibyl JP, Kosten TR, Innis RB, Gelernter J (2000) Prediction of dopamine transporter binding availability by genotype: a preliminary report. Am J Psychiatry 157:1700-1703.

Jones SR, Gainetdinov RR, Jaber M, Giros B, Wightman RM, Caron MG (1998) Profound neuronal plasticity in response to inactivation of the dopamine transporter. Proc Natl Acad Sci USA 95:4029-4034.

Karoum F, Chrapusta SJ, Egan MF (1994) 3-Methoxytyramine is the major metabolite of released dopamine in the rat frontal cortex: reassessment of the effects of antipsychotics on the dynamics of dopamine release and metabolism in the frontal cortex, nucleus accumbens, and striatum by a simple two pool model. J Neurochem 63:972-979.

Knecht S, Breitenstein C, Bushuven S, Wailke S, Kamping S, Floel A, Zwitserlood P, Ringelstein EB (2004) Levodopa: faster and better word learning in normal humans. Ann Neurol 56:20-26.

Li S, Cullen WK, Anwyl R, Rowan MJ (2003) Dopamine-dependent facilitation of LTP induction in hippocampal CA1 by exposure to spatial novelty. Nat Neurosci 6:526-531.

Lisman JE, Grace AA (2005) The hippocampal-VTA loop: controlling the entry of information into long-term memory. Neuron 46:703-713.

Lisman JE, Otmakhova NA (2001) Storage, recall, and novelty detection of sequences by the hippocampus: elaborating on the SOCRATIC model to account for normal and aberrant effects of dopamine. Hippocampus 11:551-568.

Lotta T, Vidgren J, Tilgmann C, Ulmanen I, Melen K, Julkunen I, Taskinen J (1995) Kinetics of human soluble and membrane-bound catechol $O$ methyltransferase: a revised mechanism and description of the thermolabile variant of the enzyme. Biochemistry 34:4202-4210.

Matsumoto M, Weickert CS, Akil M, Lipska BK, Hyde TM, Herman MM, Kleinman JE, Weinberger DR (2003) Catechol O-methyltransferase mRNA expression in human and rat brain: evidence for a role in cortical neuronal function. Neuroscience 116:127-137.

Mattay VS, Tessitore A, Callicott JH, Bertolino A, Goldberg TE, Chase TN, Hyde TM, Weinberger DR (2002) Dopaminergic modulation of cortical function in patients with Parkinson's disease. Ann Neurol 51:156-164.

Meyer-Lindenberg A, Kohn PD, Kolachana B, Kippenhan S, McInerney-Leo A, Nussbaum R, Weinberger DR, Berman KF (2005) Midbrain dopamine and prefrontal function in humans: interaction and modulation by COMT genotype. Nat Neurosci 8:594-596.

Mill J, Asherson P, Browes C, D'Souza U, Craig I (2002) Expression of the dopamine transporter gene is regulated by the 3' UTR VNTR: evidence from brain and lymphocytes using quantitative RT-PCR. Am J Med Genet 114:975-979.

Morrison JH, Foote SL, O'Connor D, Bloom FE (1982) Laminar, tangential and regional organization of the noradrenergic innervation of monkey cortex: dopamine-beta-hydroxylase immunohistochemistry. Brain Res Bull 9:309-319.

Mozley LH, Gur RC, Mozley PD, Gur RE (2001) Striatal dopamine transporters and cognitive functioning in healthy men and women. Am J Psychiatry 158:1492-1499.

Pezawas L, Meyer-Lindenberg A, Drabant EM, Verchinski BA, Munoz KE, Kolachana BS, Egan MF, Mattay VS, Hariri AR, Weinberger DR (2005) 5-HTTLPR polymorphism impacts human cingulate-amygdala interactions: a genetic susceptibility mechanism for depression. Nat Neurosci 8:828-834.

Piccini PP (2003) Dopamine transporter: basic aspects and neuroimaging. Mov Disord Suppl 7:S3-S8.

Otmakhova NA, Lisman JE (1996) $\mathrm{D}_{1} / \mathrm{D}_{5}$ dopamine receptor activation increases the magnitude of early long-term potentiation at CA1 hippocampal synapses. J Neurosci 16:7478-7486.

Otten LJ, Henson RN, Rugg MD (2002) State-related and item-related neural correlates of successful memory encoding. Nat Neurosci 5:1339-1344.

Schott BH, Sellner DB, Lauer CJ, Habib R, Frey JU, Guderian S, Heinze HJ, Düzel E (2004) Activation of midbrain structures by associative novelty and the formation of explicit memory in humans. Learn Mem 11:383-387.

Schröder N, O’Dell SJ, Marshall JF (2003) Neurotoxic methamphetamine regimen severely impairs recognition memory in rats. Synapse 49:89-96.

Seidenbecher CI, Smalla KH, Fischer N, Gundelfinger ED, Kreutz MR (2002) Brevican isoforms associate with neural membranes. J Neurochem 83:738-746.

Sesack SR, Hawrylak VA, Matus C, Guido MA, Levey AI (1998) Dopamine axon varicosities in the prelimbic division of the rat prefrontal cortex exhibit sparse immunoreactivity for the dopamine transporter. J Neurosci 18:2697-2708.

Siciliano R, Fornai F, Bonaccorsi I, Domenici L, Bagnoli P (1999) Cholinergic and noradrenergic afferents influence the functional properties of the postnatal visual cortex in rats. Vis Neurosci 16:1015-1028. 
Squire LR, Stark CE, Clark RE (2004) The medial temporal lobe. Annu Rev Neurosci 27:279-306.

Tulving E (2002) Episodic memory: from mind to brain. Annu Rev Psychol 53:1-25.

Tulving E, Habib R, Nyberg L, Lepage M, McIntosh AR (1999) Positron emission tomography correlations in and beyond medial temporal lobes. Hippocampus 9:71-82.

Tunbridge EM, Bannerman DM, Sharp T, Harrison PJ (2004) Catechol-omethyltransferase inhibition improves set-shifting performance and elevates stimulated dopamine release in the rat prefrontal cortex. J Neurosci 24:5331-5335.

van Oostrom I, Dollfus S, Brazo P, Abadie P, Halbecq I, Thery S, Marie RM
(2003) Verbal learning and memory in schizophrenic and Parkinson's disease patients. Psychiatry Res 117:25-34.

Vargha-Khadem F, Gadian DG, Watkins KE, Connelly A, Van Paesschen W, Mishkin M (1997) Differential effects of early hippocampal pathology on episodic and semantic memory. Science 277:376-380.

Wagner AD, Schacter DL, Rotte M, Koutstaal W, Maril A, Dale AM, Rosen BR, Buckner RL (1998) Building memories: remembering and forgetting of verbal experiences as predicted by brain activity. Science 281:1188-1191.

Wittmann BC, Schott BH, Guderian S, Frey JU, Heinze HJ, Duzel E (2005) Reward-related FMRI activation of dopaminergic midbrain is associated with enhanced hippocampus-dependent long-term memory formation. Neuron 45:459-467. 